\title{
DYSPEPSIE
}

\section{Angst ist ein wichtiger Auslöser}

- Dyspeptische Beschwerden sind ein häufiges Symptom in der Praxis. „Nicht bei jedem Patienten muss primär endoskopiert werden“, sagte Prof. Herbert Koop, Berlin. Eine endoskopische Untersuchung sei dann zwingend erforderlich, wenn Alarmsymptome wie Anämie oder Gewichtsverlust vorliegen. Ansonsten sei es durchaus sinnvoll, primär eine säurehemmende Therapie zu verordnen. „Viele Patienten sprechen gut auf eine probatorische Therapie mit Protonenpumpeninhibitoren an“, so Koop.

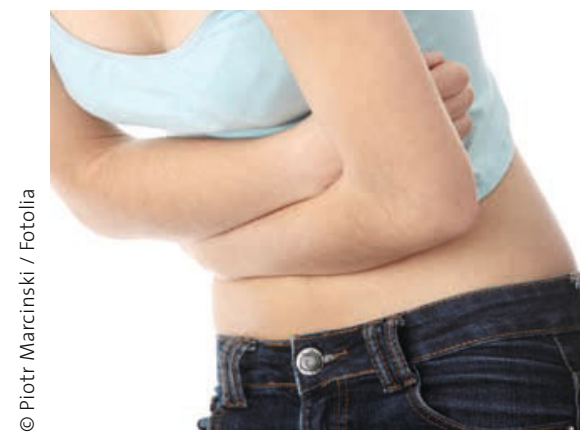

Dyspepsie ohne Alarmsymptome: probatorische PPI-Therapie häufig erfolgreich.

\section{BEHANDLUNG MIT PPI}

\section{Auch bei Schwangeren vertretbar}

- Im Allgemeinen werden Refluxbeschwerden in der Schwangerschaft zunächst mit einem Anatazidum behandelt. Reicht das nicht aus, wird eine Step-upStrategie empfohlen, d.h. es kommen $\mathrm{H}_{2}$ Blocker oder PPI zum Einsatz. In einer Metaanalyse wurden jetzt die Daten von 1530 Schwangeren, die einen PPI erhielten, analysiert. Dabei ergab sich kein erhöhtes Risiko für Missbildungen, Spontanaborte oder Frühgeburten. „Nach diesen Ergebnissen ist der Einsatz eines PPI bei Schwangeren mit antazidarefraktärer Symptomatik durchaus vertretbar", so Prof. Stephan Miehlke, Dresden. STI =

Quelle: 18. Gastroenterologie-Update-Seminar, Wiesbaden
Die hohe Wirksamkeit signalisiert, dass viele Patienten mit Dyspepsie unter einer nicht erosiven Refluxkrankheit leiden und/oder Antirheumatika bzw. Analgetika einnehmen, die die Magenschleimhaut schädigen. „Bei diesen Auslösern ist es nachvollziehbar, dass PPI eine gute Wirkung entfalten", so Koop.

Bei den Patienten, die nicht auf PPI ansprechen, muss eine schwerwiegende hypomotile Störung im Sinne einer Gastroparese diskutiert werden. In diesem Fall ist ein Therapieversuch mit einem Prokinetikum indiziert. Die Indikation für eine Endoskopie sollte bei diesen Patienten sehr großzügig gestellt werden.

Die Ergebnisse einer neuen Studie deuten darauf hin, dass Angst, jedoch nicht Depression ein wichtiger Auslöser für dyspeptische Beschweren darstellt. „Bei solchen Patienten entwickelt sich die Angst nicht als Folge der Symptome, sondern ist die zugrundeliegende pathophysiologische Störung", so Koop. STI . Quelle: XIII. Gastroenterologie-Seminarwoche, Titisee

\section{GASTROINTESTINALE BLUTUNG}

\section{Erhöhtes Risiko unter SSRI}

- Dass NSAR und ASS Magen-Darm-Blutungen verursachen können, ist hinreichend bekannt. Weniger bekannt ist das erhöhte Risiko für eine obere gastrointestinale Blutung unter Serotonin-Wiederaufnahmehemmern (SSRI). Nach einer großen Fall-Kontroll-Studie wird das Risiko fast verdoppelt. Dabei handelt es sich um einen Klasseneffekt, eine Dosisabhängigkeit konnte aber nicht nachgewiesen werden. Die gleichzeitige Einnahme von SSRI und NSAR führt zum weiteren Anstieg des Blutungsrisikos. Am stärksten gefährdet sind Patienten mit SSRI, NSAR und ASS.

STI =

Quelle: 18. Gastroenterologie-Update-Seminar, Wiesbaden 